\title{
LASERS IN DENTISTRY
}

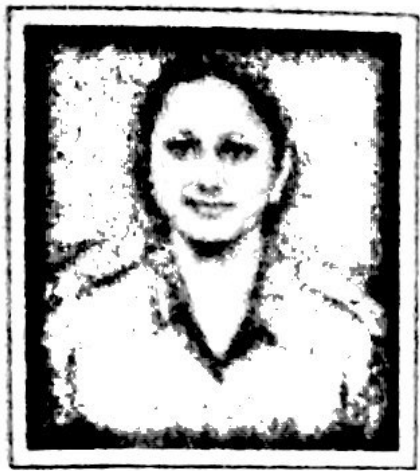

The concept of using lasers for dental treatment has been around for a number of years. Initially the attempts were not extremely successful. However, with rapid advancement in technology, dental lasers are impressive.

Materials that are used in restorations are used with a laser light source. Lasers are also used for detecting decay in teeth. No more prickly picks and shooting pains into a sensitive tooth or gum tissue.

Special lasers are used for cutting hard tissues like bone and teeth with no discomfort i.e. a pain free experience. Perfect for phobic or anxious patients.

When thinking of lasers, many of us picturise a science fiction movie, which characterizes a laser weapon capable of melting or destroying property at great distances. While certain laser technology can indeed cause this type of damage, most lasers, including those used in dentistry, are engineered and designed to perform special functions without changing or damaging the surrounding tissues or materials.
Lt. Dr. Shovana Gyawali, BDS, FAC, Dental Surgeon

\section{LASER}

L- Light A-Amplification by S-Stimulated E -Emission of R-Radiation.

\section{TYPES OF LASERS USED IN DENTISTRY}

\section{DIODE LASER}

This type of laser works best on soft tissues and can cut very precisely with little or no collateral damage depending on its settings used during the surgery.

The diode laser many times can be used without anesthesia doing very precise anterior aesthetic surgery without causing bleeding or discomfort. It is the best laser for this purpose.

\section{CO-2 LASER}

It is highly absorbed in water and is very good at cutting soft tissues without causing bleeding. It is also the best laser used to control bleeding in an area.

\section{ERBIUM LASER}

It is used in pulse mode and highly absorbed in water at very low depths. When used in hard tissues, it heats up the water within the hard tissue and causes that water to be turned into steam. This causes a mini-explosion to ${ }^{\mathrm{j}}$ occur and the hard tissue is removed.

The tissue below is not affected since the 1 laser only works at very low depths, allowing 1 precise control. This allows the dentists to 
cut hard tissue like tooth or bone without anesthesia.

It can also be used to cut soft tissue but not as precise as the diode laser and it does not control bleeding.

Laser removes a cavity without the pressure and vibration of drill. Patients will not need to have an injection or leave the dentists office with a mouth numb from lignocaine, and eliminates about 20 minutes per tooth spent waiting for the painkiller to act. It can also make a smaller hole than a drill, thus requiring a smaller filling.

\section{ANTI-BACTERIAL EFFECT}

Some research indicated a laser might kill underlying bacteria that caused the cavity, so that decay does not continue. Dentists also are studying whether lasers might one day make root canals and other surgeries less painful.

\section{LASER-FOR GUM SHAPING}

Laser can be used to work on gum tissue. Perfect for periodontal treatment and gum re-shaping and gum contouring. Lasers can also be used to treat patients with gummy smiles. In this process, laser gently removes unwanted or diseased gum tissue or remove the bacteria in periodontal pockets to promote healing, resulting in perfect smile.

\section{LASER TREATMENT + PORCELAIN VENEERS}

Laser treatments are used in conjunction with the application of porcelain veneers that do wonders for straightening smile lines and producing symmetry in a smile. This technique is popularly known as Instant orthodontics.
Apthous ulcers (mild case) can be quickly and painlessly treated with laser technology that leads to extremely rapid healing.

\section{LASERS IN COSMETIC DENTISTRY}

Laser cosmetic dentistry deals with laser removal of composite fillings, trimming of tooth structures for preparing porcelain veneer make over (instant orthodontics). Apart from these, laser treatment can also be used in treating tetracycline stains. Anything that you put in your mouth will stain your tecth like that of staining a T-shirt after pouring something on it. E.g., excessive coffee or tea drinking. In such cases, laser teeth whitening can be done.

Whitening teeth can be accomplished with special solutions that are applied to the tooth surface in the dental clinic and activated by laser energy. Colour changes of several shades are possible in a very short time. When combined with home tray based whitening systems, dramatic changes can be seen in even the most difficult cases (Tetracycline staining etc).

Patients and dentists must wear goggles during laser treatment to protect their eyes from the intense light beam.

Dental Lasers have been shown to be safe and effective for treating both children and adults. Very specific equipments and training are required to incorporate this technology into the dental patients. We look to the future with great excitement as the use of laser energy in dentistry expands to include many more procedures

\section{References websites:}

1. www.birmingham-laser-dentistry.com

2. www.losgatosdentist.com

3. www.floss.com/laser\%20dentistry.htm 